\title{
Evaluation of the QTc interval during lenvatinib treatment in radioiodine-refractory differentiated thyroid cancer: reports from the real-life clinical practice
}

\author{
Michela Marina1, Maria Francesca Serra, Paolo Del Rio² \& Graziano Ceresini*,1 \\ ${ }^{1}$ Department of Medicine \& Surgery, Unit of Internal Medicine \& Onco-Endocrinology, University of Parma, University Hospital of \\ Parma, Parma, Italy \\ ${ }^{2}$ Department of Medicine \& Surgery, Surgery Unit, University of Parma, University Hospital of Parma, Parma, Italy \\ *Author for correspondence: Tel.: +39 0521703 334; Fax: +39 052170 3307; graziano.ceresini@unipr.it
}

As for other tyrosine kinase inhibitors, a prolongation of ECG-recorded QTC intervals may be observed during lenvatinib treatment; a warning on this phenomenon has been stated. However, methods and frequency of ECG recordings have seldom been reported in this context. We present two cases of patients treated with lenvatinib for radioiodine-refractory differentiated thyroid cancer in whom the QTC interval was long monitored through a weekly 12-lead ECG registration. Overall, the maximum QTc increase above baseline was 3 and $31 \mathrm{~ms}$ in the first and second patient, respectively. QTc interval did not reach the toxicity value for drug withdrawal in either of the patients. These data may provide further information on cardiac safety profile of lenvatinib in a real-life practice.

First draft submitted: 20 February 2019; Accepted for publication: 8 July 2019; Published online: 16 August 2019

Keywords: case report $\bullet E C G \bullet$ lenvatinib $\bullet$ thyroid cancer

Lenvatinib is an oral multitarget tyrosine kinase inhibitor (TKI). Its major sites of action are represented by VEGFR1-3, RET, FGFR1-4, PDGFR- $\alpha$ and KIT [1-3].

In the Phase III, randomized, double-blind, multicenter study, SELECT, it was found that patients with radioiodine-refractory differentiated thyroid cancer (RR-DTC) treated with lenvatinib significantly prolonged progression-free survival compared with placebo [4]. These results represent a very important step in order to consider lenvatinib as a fundamental drug for RR-DTC.

The recent American Thyroid Association guidelines [5] state TKI therapy should be considered for the treatment of RR-DTC patients with metastatic, rapidly progressive, symptomatic and/or imminently threatening disease not otherwise amenable to local control by other approaches, but that patients should be counseled on the risks and benefits of TKI therapy. Among TKI-related adverse events, the prolongation of ECG-recorded QTc interval has been listed, and warning has been made on the possible occurrence of such phenomenon, especially when electrolyte modifications should concomitantly occur during such treatment. Most studies do not report QTc interval prolongation among the most frequently observed adverse events in patients with lenvatinib treatment; however, the description of ECG recording is seldom reported in those studies. On the other hand, warnings have been made by several scientific societies and medicine agencies, stating that patients should be monitored in this regard. Therefore, information is still required regarding the real-life-impact of lenvatinib on QTc interval in the follow-up of these patients.

In this study, we report data on QTc interval monitoring in two patients with RR-DTC treated with lenvatinib and followed through a weekly ECG recording. 


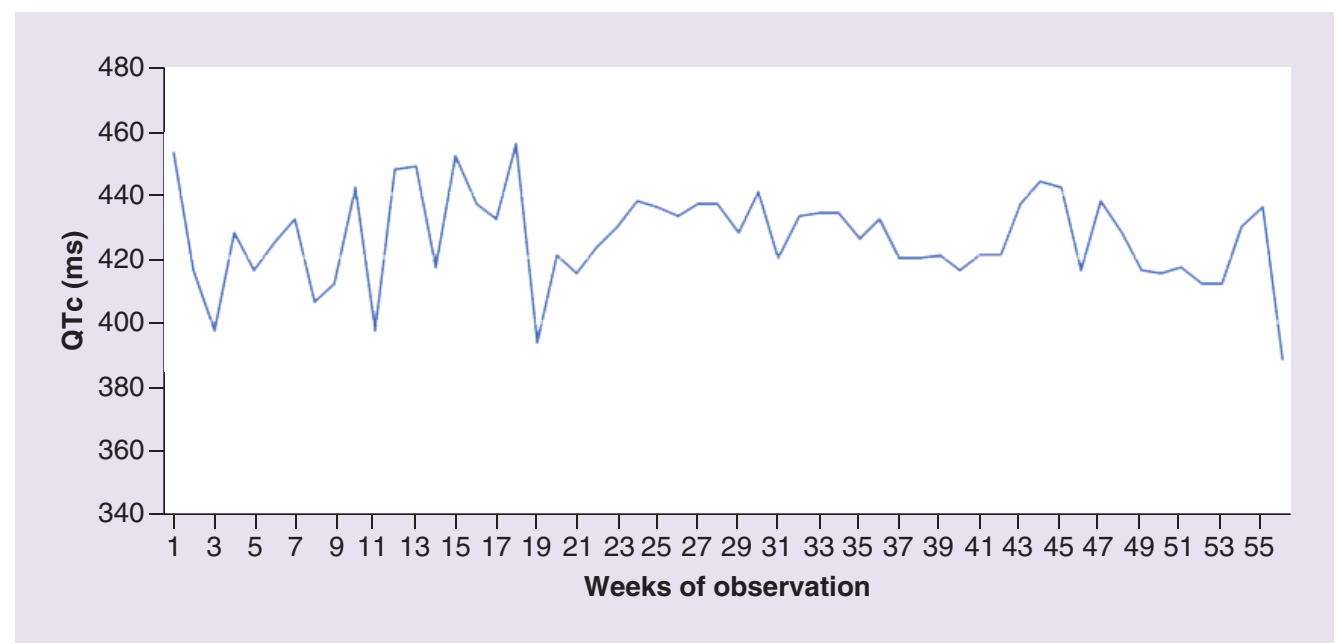

Figure 1. Values of QTc interval (ms) in patient number 1, weekly recorded during the 56 weeks of observation.

\section{Case number 1}

A 51-year-old male patient was initially evaluated in November 2012.

The history of the patient was consistent with carcinoma of the bladder, which was diagnosed 5-years earlier, treated with a partial removal of the bladder and subsequent local administration of Bacillus Calmette-Guérin. A local recurrence of the tumor was treated by a further excision.

He underwent a surgical biopsy of a mediastinal mass, which was positive for FDG-PET with the result of tall-cell papillary thyroid cancer. Three lung masses of the medial and inferior right lobe were also biopsied with the histology consistent with tall-cell variant papillary thyroid cancer. Enlargement of several mediastinal lymph nodes was found on the CT scan. A near total thyroidectomy was performed with an incomplete excision of the large mediastinal mass. The right innominate vein was found to be infiltrated. Histological diagnosis was papillary thyroid cancer with positivity for BRAF V600E mutation and for $p 53$.

The patient was submitted to $150 \mathrm{mCi}$ radioactive ${ }^{131} \mathrm{I}$ (RAI) treatment after thyroid hormone withdrawal with a post-dose whole-body scan (WBS) consistent with thyroid remnants, uptake in the mediastinum corresponding to the area of the incompletely removed mass, and uptake in the Barety's space. In 2014, a constant increase in thyroglobulin was noted together with an increment in the number and volume of lung metastases. This led to a further $200 \mathrm{mCi}$ RAI treatment. The post-dose WBS revealed one more mediastinal uptake compared with the previous WBS. In October 2014, the CT scan demonstrated enlargement of cervical lymph nodes and a volume increase of the mediastinal mass with sternum infiltration, and enlargement of several mediastinal lymph nodes; new lung nodules were also demonstrated. Zolendronic acid and fentanyl were started. In June 2015, lesions had increased in volume, especially the mediastinal mass with an increase of more than $20 \%$ compared with the previous CT findings. Despite ${ }^{131}$ I avidity, the tumor was defined as radioiodine-refractory and, according to the Response Evaluation Criteria In Solid Tumors (RECIST) version 1.1 criteria, it was defined as a progressive disease. In January 2016, a treatment with the TKI sorafenib was started with a daily dose of $800 \mathrm{mg}$. After 6 months, a CT scan revealed a stability of the disease but after 1 year, a further progression was documented with an increase in number and size of the metastatic lesions. Sorafenib was stopped and after a 1-month wash-out, a further treatment with lenvatinib was started with a dramatic improvement of the clinical status and CT scans at the 6-month follow-up. Cardiac ultrasonography and 12-lead ECG recording were performed prior to beginning lenvatinib treatment. ECG was recorded in a supine position after a 30 min rest by a MAC 1600 machine (GE Medical Systems, WI, USA). Subsequently, ECG was recorded weekly during the clinical follow-up by the same modality as described for basal recording. This led us to tightly monitor the QTc interval. The results of this QTc monitoring are demonstrated in Figure 1. The QTc interval was found to be $453 \mathrm{~ms}$ before lenvatinib was started and ranged from 388 to $456 \mathrm{~ms}$ during the 56-week observation period. Sinus rhythm was always documented. Heart rate ranged from 57 to $97 \mathrm{bpm}$. A 1-month blood sampling revealed that sodium, potassium and calcium serum concentrations were constantly within the reference range during the entire period of observation. Serum 
thyroid-stimulating hormone (TSH) was monthly monitored and was almost constantly maintained $\leq 0.1 \mathrm{mIU} / \mathrm{l}$. This required an increase in 1-thyroxine dose during the follow-up.

Echocardiographic analysis performed after 1 year of lenvatinib treatment showed normal left ventricular mass and volume with an ejection fraction $(\mathrm{EF})$ of $68 \%$. The results of cardiac ultrasound examination performed prior to lenvatinib treatment were not available.

The ACE inhibitor ramipril was also administered to treat hypertension, which was successfully controlled. The patient was also treated with alendronic acid and oxycodone, after fentanyl discontinuation, as analgesic therapy. Furosemide was also added after the diagnosis of metastatic plural effusion.

The disease was stable until the thirteenth month of lenvatinib treatment. Afterward, an increase in the size and number of metastatic lesions, especially at the lung and mediastinum, was documented with a subsequent occurrence of dysphagia, dyspnea and, eventually, cachexia. For this reason, the patient was hospitalized, and the ECG was no longer recorded weekly. Owing to this we displayed the QTc profile for the first 56 weeks of lenvatinib treatment in the results, because this was the interval in which ECG was regularly recorded weekly. The patient died because of disease progression in June 2018.

\section{Case number 2}

A 52-year-old male patient was initially evaluated in June 2016.

The history of the patient was characterized by noninsulin-dependent diabetes, hyperuricemia and hypertension. The treatment consisted of metformin, allopurinol and atenolol.

In 2003, he underwent a thyroidectomy for follicular carcinoma with multiple bone metastases. When he was referred to our unit, he had been submitted to nine RAI treatments for a total of $1810 \mathrm{mCi}^{131} \mathrm{I}$. The patient was also submitted to a surgical orthopedic procedure for stabilizing vertebral fractures. After being referred to our unit, he was operated for a loco-regional recurrence of follicular thyroid carcinoma. In February 2017, the FDG-PET and CT scans revealed an increase in the number and size of bone metastases and the occurrence of new lung and thorax soft tissue metastases. The tumor was defined as radiorefractory and a progressive disease was defined, based on the RECIST criteria. In March 2017, treatment with lenvatinib was started at $24 \mathrm{mg}$ per day. After 2 months, the dose was reduced at $14 \mathrm{mg}$ per day because of oral mucositis. A significant relief was obtained, and the treatment was maintained at that dose. A temporary discontinuation of treatment was observed for dental surgery. Anti-hypertensive therapy was modified because of poor blood pressure control and amlodipine, telmisartan and hydrochlorothiazide were started with good blood pressure control.

Weekly 12-lead-ECG recordings were started immediately before lenvatinib treatment with the same modalities as described for patient \#1. The QTc interval was found to be $434 \mathrm{~ms}$ before lenvatinib was started and ranged from 389 to 465 ms during the 65-week observation period (Figure 2). Sinus rhythm was always documented. Heart rate ranged from 59 to $111 \mathrm{bpm}$ during the whole period of observation.

The echocardiographic analysis performed before the beginning of lenvatinib treatment showed normal left ventricular mass and volume with an EF of 65\%. Sectal hypertrophy $(14 \mathrm{~mm})$ was documented. After 1 year of lenvatinib treatment, the $\mathrm{EF}$ was $60 \%$ with the remaining parameters being comparable with those documented at the first echocardiographic analysis performed prior to lenvatinib treatment.

A 1-month blood sampling performed during the treatment revealed that sodium, potassium and calcium serum concentrations were constantly within the reference range. Serum TSH was monitored every month and was almost constantly $\leq 0.1 \mathrm{mIU} / \mathrm{l}$, provided the dose of l-thyroxin was increased during the follow-up. The patient is alive with a stable disease, and lenvatinib is still maintained at $14 \mathrm{mg}$ per day.

\section{Discussion}

The data from the present case reports demonstrate the lack of critical prolongation of the QTc interval during lenvatinib treatment in quite narrow intervals of evaluation.

TKIs are associated with important side effects, which may require a modification or interruption of treatment and, for some cases, drug withdrawal. The prevention of TKI toxicities is therefore mandatory and is principally brought about by clinical, laboratory and instrumental patient monitoring.

The most important cardiac side effect is represented by the prolongation of the electrocardiographic QTc interval [6].

The ECG-recorded QT interval gives information on the total duration of ventricular activation and recovery [6]. The International Conference on Harmonisation of Technical Requirements for Registration of Pharmaceuticals 


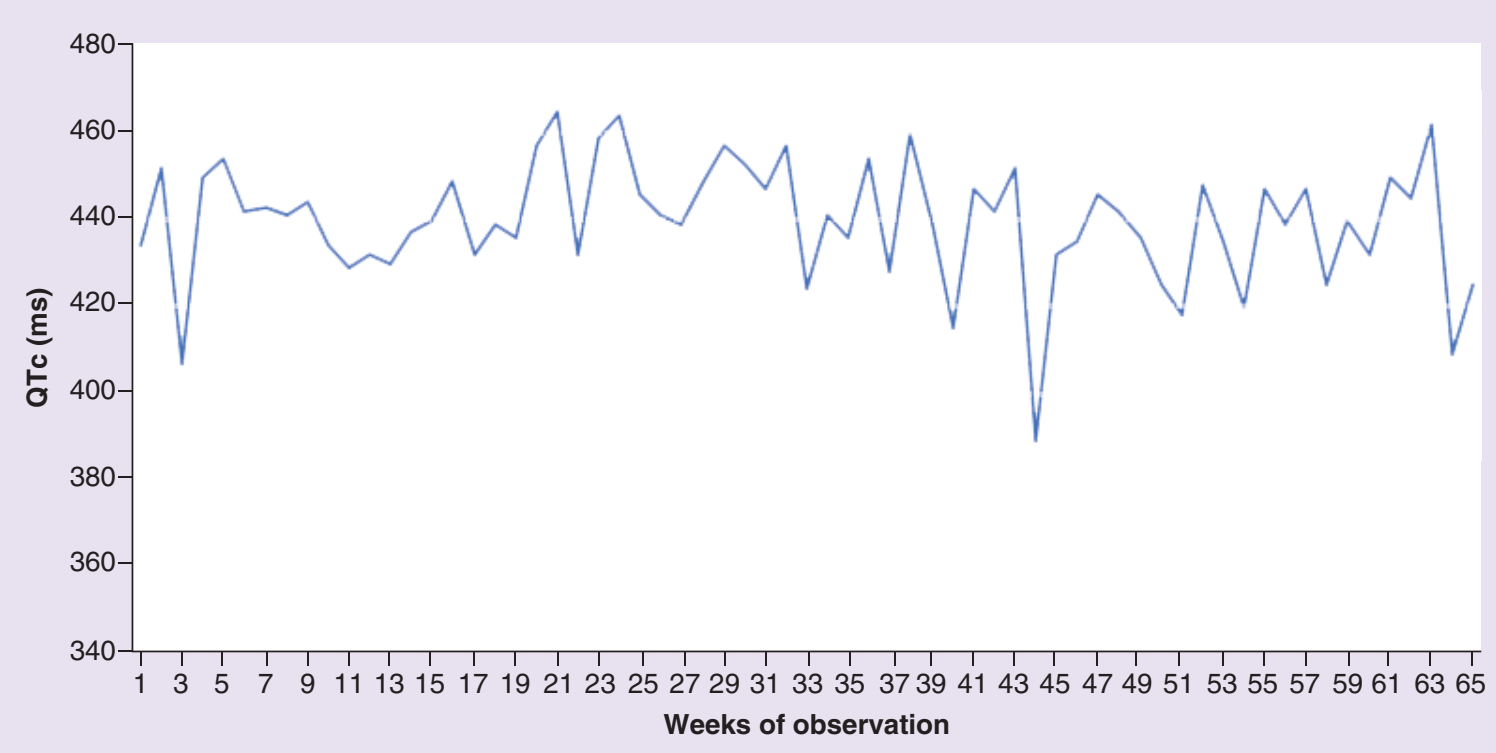

Figure 2. Values of QTc interval (ms) in patient number 2, weekly recorded during the 65 weeks of observation.

for Human Use considers QTc prolongation $>500 \mathrm{~ms}$ (and deltaQT [change from baseline] of $>60 \mathrm{~ms}$ ) to be of particular concern because torsades de pointes may arise in this condition, whereas it rarely occurs when QTc is $<500 \mathrm{~ms}$ [7]. A Phase III trial has shown a QTc prolongation in $8 \%$ of patients affected by medullary thyroid carcinoma treated with vandetanib [8]. In addition, cabozantinib and ceritinib have been shown to induce a mild to moderate degree of QTc interval prolongation [9]. No QTc prolongation has been listed among the adverse events in a Phase II trial of lenvatinib in RR-DTC [10]. Similarly, other studies do not report QTc prolongation among the most frequent side effects during lenvatinib treatment [11-14] and considering cardiovascular adverse events, grade 3 (according to the adverse events scale) hypertension has been shown to represent the principal reason for early lenvatinib dose reduction in a retrospective study [15]. In the Phase III SELECT study on lenvatinib treatment in RR-DTC, QTc prolongation was found to occur in 8 and $1.5 \%$ of patients when any grade or a grade $\geq 3$ were considered, respectively [4]. Nevertheless, although with a low-frequency occurrence, this phenomenon still remains a life-threatening event and warnings have been made in this regard [16], including an indication for ECG and electrolyte monitoring should be considered at baseline, 7 and 15 days after initiation of a TKI or dose changes, monthly during the first 3 months and then periodically during treatment depending on patient status [17]. A general recommendation from the US FDA and EMA is to temporarily interrupt treatment in case of QTc prolongation above $500 \mathrm{~ms}$ (or if QTc prolongation is $>60 \mathrm{~ms}$ above baseline). In this case, electrolyte abnormalities should be corrected and cardiac risk factors for QT prolongation controlled [18]. Treatment can then be resumed at a reduced dose once the QTc normalizes [17]. In this context, it is important to note that, overall, the maximum QTc increases above baseline we observed in our patients were 3 and $31 \mathrm{~ms}$ in the first and second patient, respectively. QTc interval did not reach the toxicity value for drug withdrawal in either of the patients.

To our knowledge, there are no reports on QTc interval during ECG monitoring in lenvatinib patients through a weekly recording for such a long period of observation, as we did in our study. In this context, the lack of critical prolongation of QTc over a frequent recording could add more information on such adverse effects of lenvatinib in a real-life practice. In fact, most of the data on lenvatinib side effects come from clinical trials with patients who, based on trial selection, may be clinically different from those routinely seen. Interestingly enough, our data agree with other reports demonstrating the lack of QTc prolongation at high plasma lenvatinib concentrations obtained after a single dose of $32 \mathrm{mg}$ in healthy volunteers [19].

Patient number 1 had been treated with sorafenib for almost 1 year before the beginning of lenvatinib. We do not have data on the QTc recording during such treatment and we therefore cannot compare the effects of these two TKIs on QTc interval in this patient. The same patient had been submitted to echocardiography analysis elsewhere before the beginning of lenvatinib treatment and for some unknown reasons, that report was not available. However, 
after 1 year of lenvatinib treatment, echocardiography revealed a normal EF and no significant echocardiographic abnormalities. Interestingly, the QTc interval was found to be within the range even at the recording performed during a hospital observation a few days before the fatal event.

In patient number 2, echocardiographic parameters were comparable in the recordings made prior to and after lenvatinib treatment. Particularly, the EF did not substantially change, although a lower value was noted after 65 weeks of lenvatinib treatment. In a 2015 meta-analysis, Ghataliaa et al. reported an all-grade congestive heart failure in $2.39 \%$ of cancer patients receiving VEGFR TKIs versus $0.75 \%$ patients in the non-TKI group. Highgrade congestive heart failure was reported in $1.19 \%$ patients receiving VEGFR TKIs and $0.65 \%$ of patients in the non-TKI group. However, in that study, lenvatinib was not considered [20] and although cardiac dysfunction has been reported with the use of afatinib, dabrafenib, lenvatinib, ponatinib and trametinib [8], heart failure is not commonly reported among the most frequent adverse events of lenvatinib. The importance of a relationship between TKIs and chronic heart failure is indeed emphasized in a recent metanalysis [21]; moreover, a $>20 \%$ reduction in EF among patients on lenvatinib treatment has been reported, although in a small percentage of cases [9]. These data support the need for a continuous monitoring and reassessment of cardiac function in patients treated with lenvatinib.

In conclusion, we have reported two cases of patients with RR-DTC in whom a weekly ECG recording for 56-65 weeks did not show any critical QTc prolongation. This QTc monitoring may substantially represent the effects of lenvatinib on QTc interval in the real-world clinical practice and suggest that the frequency of ECG monitoring in these patients could be prolonged with respect to the interval observed in the present cases. Overall, based on our data, it could be reasonable to hypothesize that a monthly ECG monitoring would likely be sufficient to diagnose any potential impact of lenvatinib on QTc.

These results may be useful in order to gain further information on the lenvatinib cardiological safety profile. Although our data further support the notion that QTc prolongation represents an uncommon adverse event in patients treated with lenvatinib, careful monitoring of these patients should be observed to prevent this phenomenon, including electrolyte control and the avoidance of drugs of a proven or potential impact on QTc interval.

\section{Executive summary}

- As for other tyrosine kinase inhibitors, a prolongation of ECG-recorded QTc intervals may be observed during lenvatinib treatment; a warning on this phenomenon has been stated.

- Methods and frequency of ECG recordings have seldom been reported in this context.

- The Author reported two cases of patients with radioiodine-refractory differentiated thyroid cancer in whom a weekly ECG recording for 56-65 weeks did not show any critical QTc prolongation.

- Based on the literature and of this experience the author speculate that a monthly ECG monitoring would likely be sufficient to diagnose any potential impact of lenvatinib on QTc.

Financial \& competing interests disclosure

The authors have no relevant affiliations or financial involvement with any organization or entity with a financial interest in or financial conflict with the subject matter or materials discussed in the manuscript. This includes employment, consultancies, honoraria, stock ownership or options, expert testimony, grants or patents received or pending, or royalties.

Editorial assistance was provided by A Shah and L Giacomelli (Polistudium SRL, Milan, Italy), on behalf of Content Ed Net. This assistance was supported by Eisai.

Informed consent

Informed consent was obtained from patients to collect data for scientific publications concerning their cases.

Open access

This work is licensed under the Attribution-NonCommercial-NoDerivatives 4.0 Unported License. To view a copy of this license, visit http://creativecommons.org/licenses/by-nc-nd/4.0 


\section{References}

Papers of special note have been highlighted as: $\bullet$ of interest; $\bullet \bullet$ of considerable interest

1. Matsui J, Yamamoto Y, Funahashi Y et al. E7080, a novel inhibitor that targets multiple kinases, has potent antitumor activities against stem cell factor producing human small cell lung cancer H146, based on angiogenesis inhibition. Int. J. Cancer 122, 664-671 (2008).

2. Matsui J, Funahashi Y, Uenaka T, Watanabe T, Tsuruoka A, Asada M. Multi-kinase inhibitor E7080 suppresses lymph node and lung metastases of human mammary breast tumor MDA-MB-231 via inhibition of vascular endothelial growth factor-receptor (VEGF-R) 2 and VEGF-R3 kinase. Clin. Cancer Res. 14, 5459-5465 (2008).

3. Okamoto K, Kodama K, Takase K et al. Antitumor activities of the targeted multi-tyrosine kinase inhibitor lenvatinib (E7080) against RET gene fusion-driven tumor models. Cancer Lett. 340, 97-103 (2013).

4. Schlumberger M, Tahara M, Wirth LJ et al. Lenvatinib versus placebo in radioiodine-refractory thyroid cancer. N. Engl. J. Med. 372 , 621-630 (2015).

-• Gives evidence for lenvainib efficacy in radioiodine-refractory thyroid cancer.

5. Haugen BR, Alexander EK, Bible KC et al. American Thyroid Association management guidelines for adult patients with thyroid nodules and differentiated thyroid cancer: the American Thyroid Association guidelines task force on thyroid nodules and differentiated thyroid cancer. Thyroid 26(1), 1-133 (2016).

- This is an evidence-based guideline relevant for practice.

6. Ghatalia P, Je Y, Kaymakcalan MD, Sonpavde G, Choueiri TK. QTc interval prolongation with vascular endothelial growth factor receptor tyrosine kinase inhibitors. Br. J. Cancer 112, 296-305 (2015).

7. Lenihan DJ, Kowey PR. Overview and management of cardiac adverse events associated with tyrosine kinase inhibitors. Oncologist 18(8), 900-908 (2013).

8. Wells SA Jr, Robinson BG, Gagel RF et al. Vandetanib in patients with locally advanced or metastatic medullary thyroid cancer: a randomized, double-blind Phase III trial. J. Clin. Oncol. 30(2), 134-141 (2012).

9. Shah RR, Morganroth J. Update on cardiovascular safety of tyrosine kinase inhibitors: with a special focus on QT interval, left ventricular dysfunction and overall risk/benefit. Drug Saf. 38, 693 (2015).

10. Cabanillas ME, Schlumberger M, Jarzab B et al. A Phase II trial of lenvatinib (E7080) in advanced, progressive, radioiodine-refractory, differentiated thyroid cancer: a clinical outcomes and biomarker assessment. Cancer 121(16), 2749-2756 (2015).

- Evidence for efficacy of lenvatinib.

11. Haddad RI, Schlumberger M, Wirth LJ et al. Incidence and timing of common adverse events in lenvatinib-treated patients from the SELECT trial and their association with survival outcomes. Endocrine 56, 121-128 (2017).

12. Berdelou A, Borget I, Godbert $\mathrm{Y}$ et al. Lenvatinib for the treatment of radioiodine-refractory thyroid cancer in real-life practice. Thyroid 28, 72-78 (2018).

13. Robinson B, Schlumberger M, Wirth LJ et al. Characterization of tumor size changes over time from the Phase III study of lenvatinib in thyroid cancer. J. Clin. Endocrinol. Metab. 101(11), 4103-4109 (2016).

14. Zhu C, Ma X, Hu Y et al. Safety and efficacy profile of lenvatinib in cancer therapy: a systematic review and meta-analysis. Oncotarget 7(28), 44545-44557 (2016).

15. Suyama K, Tomiguchi M, Takeshita $\mathrm{T}$ et al. Factors involved in early lenvatinib dose reduction: a retrospective analysis. Med. Oncol. 35(3), 19 (2018).

16. Matrone A, Valerio L, Pieruzzi L et al. Protein kinase inhibitors for the treatment of advanced and progressive radiorefractory thyroid tumors: from the clinical trials to the real life. Best Pract. Res. Clin. Endocrinol. Metab. 31(3), 319-334 (2017).

17. Zamorano JL, Lancellotti P, Muñoz DR et al. ESC position paper on cancer treatments and cardiovascular toxicity developed under the auspices of the ESC Committee for Practice Guidelines. Eur. Heart J. 37(36), 2768e801 (2016).

18. Resteghini C, Cavalieri S, Galbiati D et al. Management of tyrosine kinase inhibitors (TKI) side effects in differentiated and medullary thyroid cancer patients. Best Pract. Res. Clin. Endocrinol. Metab. 31, 349-361 (2017).

19. Shumaker RC, Zhou M, Ren M et al. Effect of lenvatinib (E7080) on the QTc interval: results from a thorough QT study in healthy volunteers. Cancer Chemother. Pharmacol. 73(6), 1109-1117 (2014).

20. Ghataliaa P, Morganb CJ, Jec Y et al. Congestive heart failure with vascular endothelial growth factor receptortyrosine kinase inhibitors. Crit. Rev. Oncol. Hematol. 94, 228-237 (2015).

21. Qi W-X, Shen Z, Tang L-N, Yao Y. Congestive heart failure risk in cancer patients treated with vascular endothelial growth factor tyrosine kinase inhibitors: a systematic review and meta-analysis of 36 clinical trials. Br. J. Clin. Pharmacol. 78, 748-762 (2014). 